\title{
Erratum to: What is the Significance of the In Transit or Interval Sentinel Node in Melanoma?
}

Jonathan S. Zager, MD, Christopher A. Puleo, PA-C, and Vernon K. Sondak, MD

Department of Cutaneous Oncology, Moffitt Cancer Center, Tampa, FL

ERRATUM TO: ANN SURG ONCOL

(2011) 18:3232-3234

DOI 10.1245/S10434-011-1996-5

In the original publication of this editorial, Fig. 1 was not correctly displayed. It is reprinted here correctly.

The online version of the original article can be found under doi:10.1245/s10434-011-1996-5.

(C) Society of Surgical Oncology 2011

Published Online: 3 November 2011

J. S. Zager, MD

e-mail: jonathan.zager@moffitt.org 

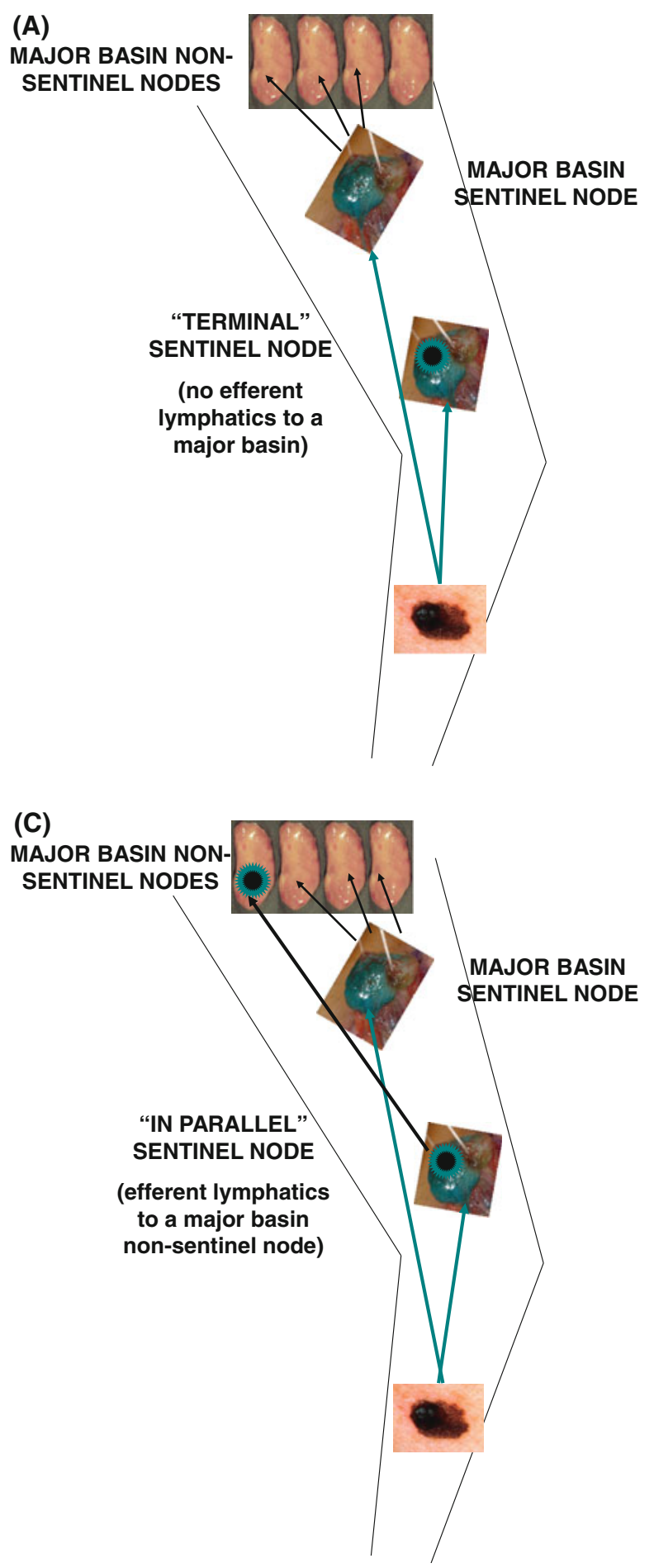

FIG. 1 Potential drainage pathways for sentinel lymph nodes situated outside of major basins and their clinical implications. a "Terminal" nodes are nodes that receive afferent lymphatics from a cutaneous primary but do not have efferent lymphatics to a major basin; if the major basin sentinel node is negative, no regional lymphadenectomy would be indicated even if a terminal sentinel node contains tumor (black circle). b "In series" nodes receive afferent lymphatics from a cutaneous primary and send efferent lymphatics to a sentinel node in the major basin; if the major basin sentinel node is negative, no regional lymphadenectomy would be indicated even if the in transit sentinel node contains tumor. c "In parallel" nodes

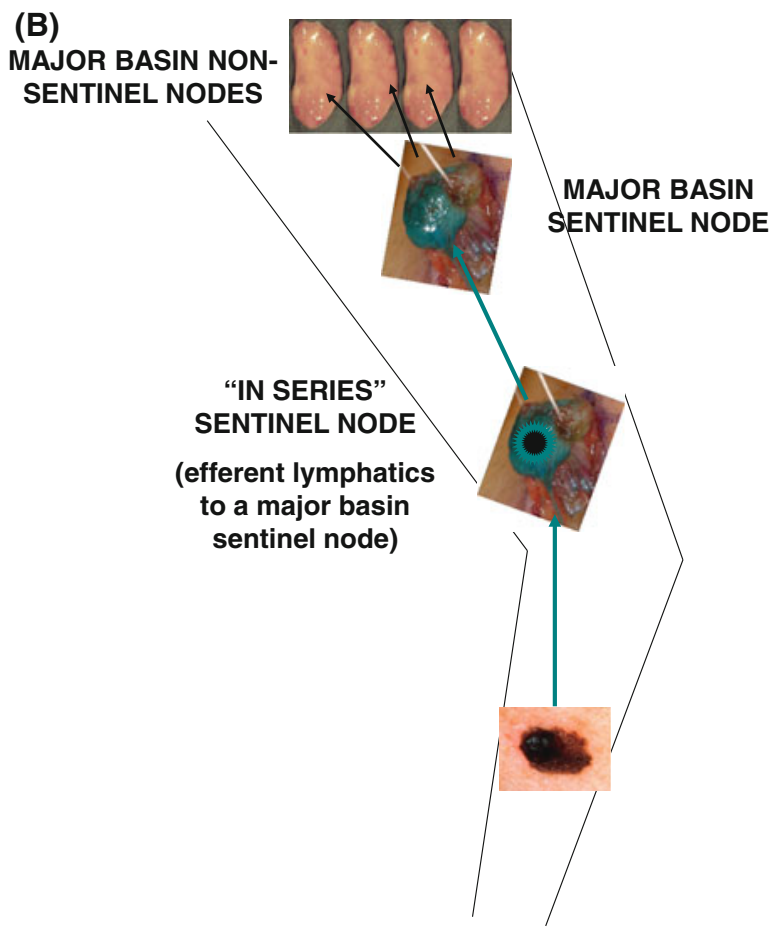

receive afferent lymphatics from a cutaneous primary but send efferent lymphatics to a non-sentinel node in the major basin; if a major basin sentinel node is negative, there would still be a substantial risk of metastatic disease within that major basin and lymphadenectomy would be appropriate to consider. (Blue lines designate afferent lymphatics containing blue dye injected at the primary site; black lines represent efferent lymphatics to secondechelon or "non-sentinel" nodes. In this representation, the primary cutaneous melanoma is situated on the lower leg and the in transit node is in the lower thigh, and the major basin nodes are in the groin.) 